\title{
Heterogeneity of Moraxella isolates found in the nasal cavities of piglets
}

Sergi López-Serrano ${ }^{1}$, Nuria Galofré-Milà', Mar Costa-Hurtado ${ }^{1}$, Ana M. Pérez-de-Rozas ${ }^{1}$ and Virginia Aragon ${ }^{1,2^{*}}$ (D)

\begin{abstract}
Background: Previous studies have shown that the genus Moraxella is commonly present in the nasal microbiota of swine.

Results: In this study, 51 isolates of Moraxella were obtained from nasal swabs from 3 to 4 week old piglets, which represented 26 different fingerprintings by enterobacterial repetitive intergenic consensus (ERIC)-PCR. Whole 165 rRNA gene sequencing allowed the identification at species level of the Moraxella spp. isolates. The majority of the field strains were identified as Moraxella pluranimalium, but Moraxella porci was also detected. In addition, a cluster of 7 strains did not group with any described Moraxella species, probably representing a new species. Subsequent phenotypic characterization indicated that strains of Moraxella pluranimalium were mainly sensitive to serum complement, while the cluster representing the putative new species was highly resistant. Biofilm formation capacity was very variable among the Moraxella spp. isolates, while adherence to epithelial cell lines was similar among selected strains. Additionally, variability was also observed in the association of selected strains to porcine alveolar macrophages. Antimicrobial tests evidenced the existence of multidrug-resistance in the strains.

Conclusions: In summary, phenotypic characterization revealed heterogeneity among Moraxella strains from the nasal cavity of piglets. Strains with pathogenic potential were detected as well as those that may be commensal members of the nasal microbiota. However, the role of Moraxella in porcine diseases and health should be further evaluated.
\end{abstract}

Keywords: Swine, Nasal microbiota, Virulence, Bacterial diversity, Moraxella

\section{Background}

Moraxella is a microbiota member of the upper respiratory tract in vertebrates, but some species may cause opportunistic infections. Best-known Moraxella infections are produced by Moraxella catarrhalis, which causes respiratory infections, including pneumonia and otitis in human [1]. In livestock, Moraxella bovis is well-known as the etiological agent of infectious keratoconjunctivitis in cattle [2]. In swine, the genus Moraxella has representative species, such as Moraxella porci, which was discovered and isolated from meninges [3] and Moraxella pluranimalium, described from an abdominal cavity isolate [4], but the

\footnotetext{
* Correspondence: virginia.aragon@irta.es

'IRTA, Centre de Recerca en Sanitat Animal (CReSA, IRTA-UAB), Campus de la Universitat Autònoma de Barcelona, 08193 Bellaterra, Spain

${ }^{2} \mathrm{OIE}$ Collaborating Centre for the Research and Control of Emerging and Re-emerging Swine Diseases in Europe (IRTA-CReSA), Bellaterra, Barcelona, Spain
}

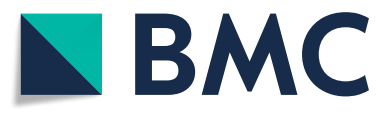

(- The Author(s). 2020 Open Access This article is distributed under the terms of the Creative Commons Attribution 4.0 International License (http://creativecommons.org/licenses/by/4.0/), which permits unrestricted use, distribution, and

reproduction in any medium, provided you give appropriate credit to the original author(s) and the source, provide a link to the Creative Commons license, and indicate if changes were made. The Creative Commons Public Domain Dedication waiver (http://creativecommons.org/publicdomain/zero/1.0/) applies to the data made available in this article, unless otherwise stated. lished. Nonetheless, Moraxella may play a role in swine as a member of the nasal microbiota, since it has been detected as one of the most abundant genera in the nasal cavity of weaned piglets [5], is abundant in the nasal microbiota of slaughter age pigs [6] and can be found in environmental samples in farrowing buildings [7].

The microbiota plays an important role in host health and disease through different mechanisms, such as maturation of the immune system, improvement of the mucosal barrier and resistance against pathogens [8]. In the case of the nasal microbiota, early colonizers can determine the stability and composition of bacterial community leading to a healthier status, as reported for children early colonized by high abundance of Moraxella, which was associated with fewer respiratory infections [9]. However, the specific role of Moraxella, among other residents of the respiratory microbiota, in health is still 
unknown in pigs. In this study, we performed a genotypic and phenotypic characterization of 51 Moraxella field isolates from the nasal turbinates of weaned piglets. We assessed their capacity of evasion of the innate immunity by examining their complement susceptibility and macrophage association capacity, as well as other characteristics closely related to its ecological niche, such as biofilm formation, mucin adhesion and cell adherence. Our results showed genotypic and phenotypic heterogeneity among the Moraxella isolates.

\section{Results}

\section{Bacterial identification and genotyping}

Initial identification of bacterial isolates by partial $16 \mathrm{~S}$ rRNA gene sequencing showed a total collection of 51 Moraxella spp. isolates. Genotyping by enterobacterial repetitive intergenic consensus (ERIC)-PCR determined 26 different ERIC fingerprintings, which were not shared among farms (Table 1). Up to 7 different fingerprinting profiles were isolated from the same farm, and up to 3 from a single piglet. One isolate of each fingerprinting (from now on referred as different strains for clarity throughout the text) were selected for further analysis. Sequencing of approximately $1360 \mathrm{bp}$ of the $16 \mathrm{~S}$ rRNA gene allowed a more precise identification of the Moraxella isolates. Sequences from the nasal isolates and sequences from Moraxella sp. type strains from the Ribosomal database were used to build an UPGMA (unweighted pair group method with arithmetic mean) tree (Fig. 1). Sixteen nasal strains clustered with more than $99.5 \%$ identity with Moraxella pluranimalium, while one strain, LL-3, was a bit more divergent, with $99.4 \%$ identity. Strain EJ45-1

Table 1 Sampled farms and nasal Moraxella spp. isolated from piglets at weaning (3-4 weeks of age). Ten piglets representing 5 litters from each farm were sampled. Name of strain indicates farm of origin, followed by piglet number and finally the number of isolate. Exceptions to this are LL and CR isolates, which indicate only the number of isolate

\begin{tabular}{|c|c|c|c|c|}
\hline Farm & Selected strains & Isolates with same ERIC PCR profile & Health status & Antimicrobial treatment $^{a}$ \\
\hline \multirow[t]{5}{*}{$\overline{G M}$} & GM3-2 & GM4-1 & Healthy & AMOX \\
\hline & GM8-1 & - & & \\
\hline & GM5-1 & GM7-2, GM9-1, GM10-1 & & \\
\hline & GM5-5 & GM7-6 & & \\
\hline & GM5-7 & GM7-8, GM7-9, GM8-4 & & \\
\hline$A R$ & AR-5A & AR-5C & Healthy & AMOX \\
\hline \multirow[t]{3}{*}{ EJ } & EJ43-3A & - & Glässer's disease & ENR \\
\hline & EJ44-2A & - & & \\
\hline & EJ45-1 & EJ45-5 & & \\
\hline \multirow[t]{5}{*}{ UK/LG } & UK1-12 & UK1-32, LG1-4, LG1-6, LG2-2, LG3-7 & Healthy & - \\
\hline & UK1-20 & LG5-5, LG8-3 & & \\
\hline & LG6-2 & - & & \\
\hline & LG6-4 & LG6-6 & & \\
\hline & LG6-7 g & - & & \\
\hline \multirow[t]{3}{*}{$C R$} & CR-18 & - & Healthy & AMOX \\
\hline & CR-10 & - & & \\
\hline & CR-7A & CR-12 & & \\
\hline KD & KD4-7 & - & Healthy & - \\
\hline \multirow[t]{7}{*}{ VL } & VL6-4 & - & Healthy & TUL, CEFT \\
\hline & VL9-7 & VL9-8, VL10-1, VL10-2 & & \\
\hline & VL6-6 & - & & \\
\hline & VL1-4 & VL1-7, VL5-3 & & \\
\hline & VL3-9 & - & & \\
\hline & VL2-5 & - & & \\
\hline & VL1-5 & VL1-6 & & \\
\hline LL & LL-3 & - & Nervous signs & Unknown \\
\hline
\end{tabular}




\section{Similarity (\%)}

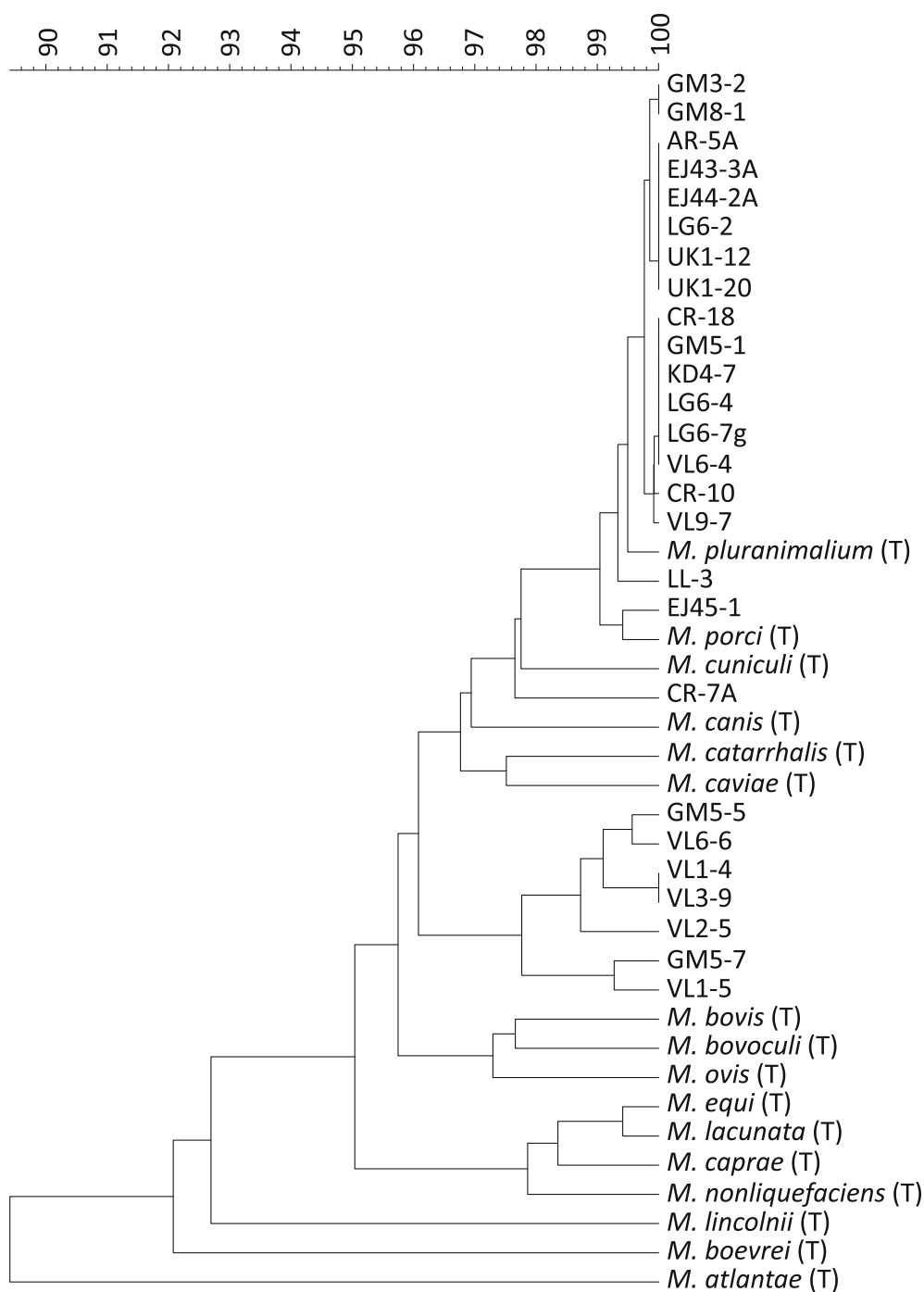

Fig. 1 UPGMA tree built with the 16S rRNA gene sequences of the Moraxella spp. isolates from the nasal cavities of piglets at weaning. Type strains (T) of different Moraxella species were included in the analysis

clustered with Moraxella porci, with $99.2 \%$ identity. Strain CR-7A showed similarity to M. porci and Moraxella cuniculi, but did not clearly cluster with any of them. Finally, a group of 7 nasal strains showed homology among them of more than $98 \%$, but did not clustered with any of the already described Moraxella species, indicating that they may represent a new species. Biochemical characterization of representative isolates can be found in Additional file 1.

\section{Antimicrobial susceptibility}

Moraxella strains from nasal microbiota showed high diversity in antibiotic susceptibility (Table 2). In general, all the strains were sensitive to amoxicillin+clavulanate and florfenicol, in contrast to trimetoprim + sulfonamide and tetracycline, where we found a high resistance rate. Other antimicrobials with low resistance rates were ceftiofur, gentamicin and colistin. Six strains showed resistance to amoxicillin, which was abolished with the $\beta$-lactamase inhibitor clavulanate. M. pluranimalium UK1-12 and UK1-20 showed only resistance to tetracycline; both strains were isolated from the same farm, which did not use antimicrobials before the sampling time. Six strains were resistant to 2 different antimicrobials. Multidrug-resistant (MDR), defined as resistance to 3 or more classes of antimicrobials, was found in 18 out of the 26 strains. A higher percentage of MDR strains was observed in the farms using antimicrobials than in those not using these drugs during lactation ( $75 \%$ versus $50 \%$, respectively), although no statistical significance was found. 


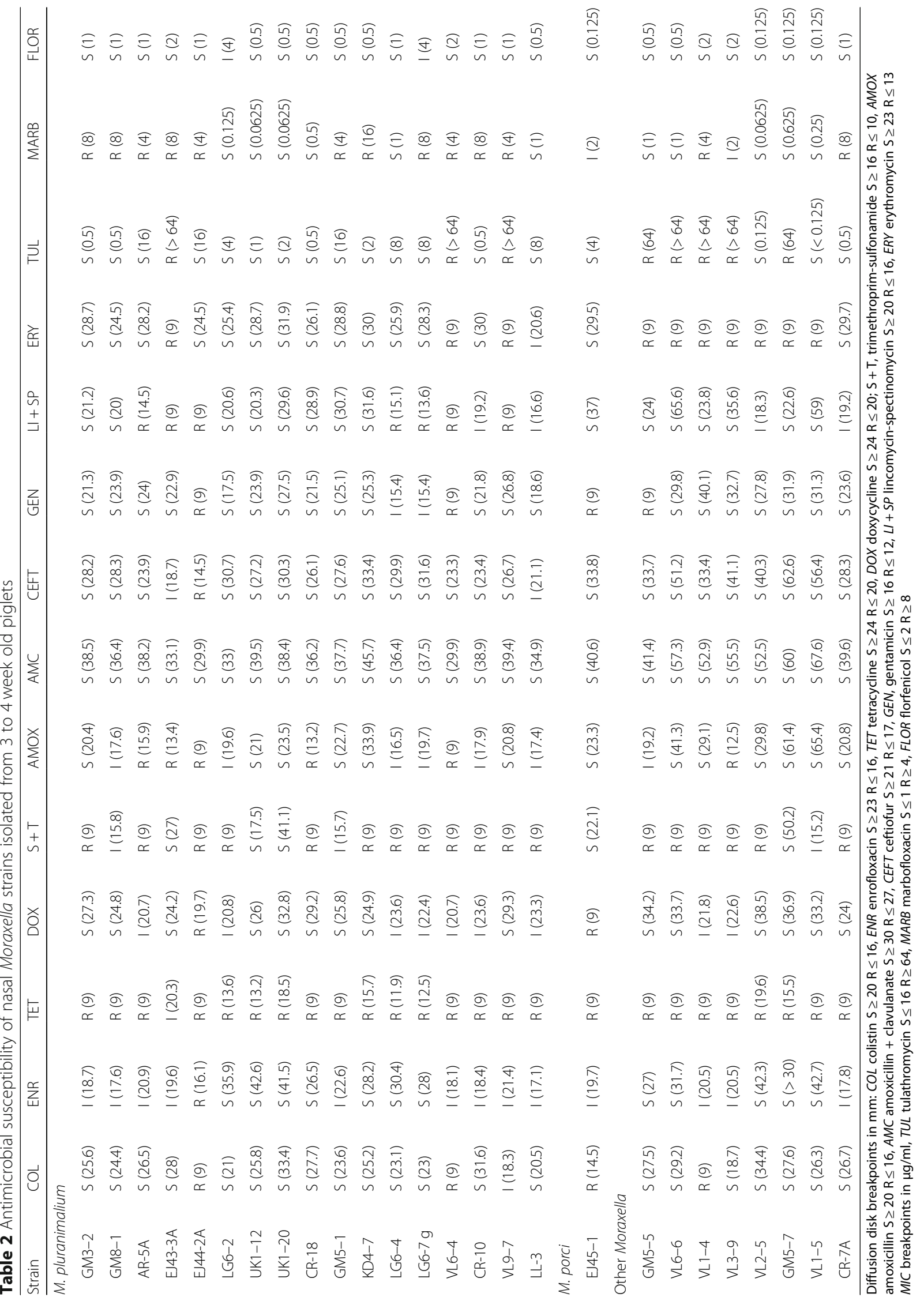




\section{Serum resistance}

Initially, bacterial suspensions were prepared in PBS obtaining irregular results, due to auto-agglutination by some strains. To overcome this problem, all the strains were resuspended in 20\% glycerol in PBS and passed through a needle if necessary; this process did not affect the viability of the bacteria (not shown).

When the strains were assayed for survival after incubation with fresh rabbit serum, 17 of the 26 (65\%) strains showed a reduction in viability of more than 3 logarithms after incubation with serum (Fig. 2). Within M. pluranimalium, 12 out of the 16 strains (75\%) showed a reduction of viability of more than 3 logs, similar to the type strain of this species, while the rest 4 (25\%) showed a reduction of 2-3 logs. On the other hand, only 2 of the 7 (29\%) strains from the cluster representing a putative new Moraxella species showed a reduction of more than 3 logs in viability, while the rest of strains in this cluster $(5 / 7 ; 71 \%)$ showed a reduction of viability of less than 2 logarithms (Fig. 2), indicating resistance to the serum complement. Moreover, strains showing the highest resistance to serum were observed within the isolates from the cluster of the putative new Moraxella species, and belonged to the same farm (VL1-4, VL3-9 and VL1-5; Fig. 2). M. porci type strain SN9-4 M, as well as strain M. porci EJ45-1, showed high susceptibility to the treatment with rabbit sera (Fig. 2).

\section{Biofilm assays}

In an attempt to select non-agglutinating variants, we performed several passes of the agglutinating strains UK1-20 and VL6-6 in broth culture. Each passage was performed with bacteria taken from the surface of the culture that did not agglutinate. Curiously, a thick biofilm was observed in the culture tubes of UK1-20 after 27 passes (UK1-20p27); the bacteria in these cultures did not agglutinate but stuck to the glass. In the case of VL6-6, a biofilm in the airliquid interphase was observed after 27 passes (VL6-6p27) (Fig. 3). This observation prompted us to examine the natural level of biofilm formation capacity of the rest of the nasal Moraxella sp. strains. We found different rates of biofilm formation that were not dependent on the Moraxella species. Observing the results, different levels of biofilm formation could be arbitrarily established: production of biofilm when Abs590 $\geq 0.6$ and of strong biofilm when $\mathrm{Abs}_{590}>1.5$. With those definitions, strains showing biofilm production capacity were $M$. pluranimalium GM3-2, GM8-1, EJ44-2A, LG6-2,, UK1-20, GM5-1, VL6-4 and VL9-7; Moraxella sp. LL-3; Moraxella sp. (putative new species cluster) VL6-6; and also the variants UK1-20p27 and VL6-6p27 used as controls (Fig. 4). Few isolates ( $M$ pluranimalium GM3-2 and LG6-2, and Moraxella sp. LL-3) were able to form strong biofilms (Fig. 4). In general, strains forming biofilm under static conditions formed biofilm also under shaking conditions, although at different

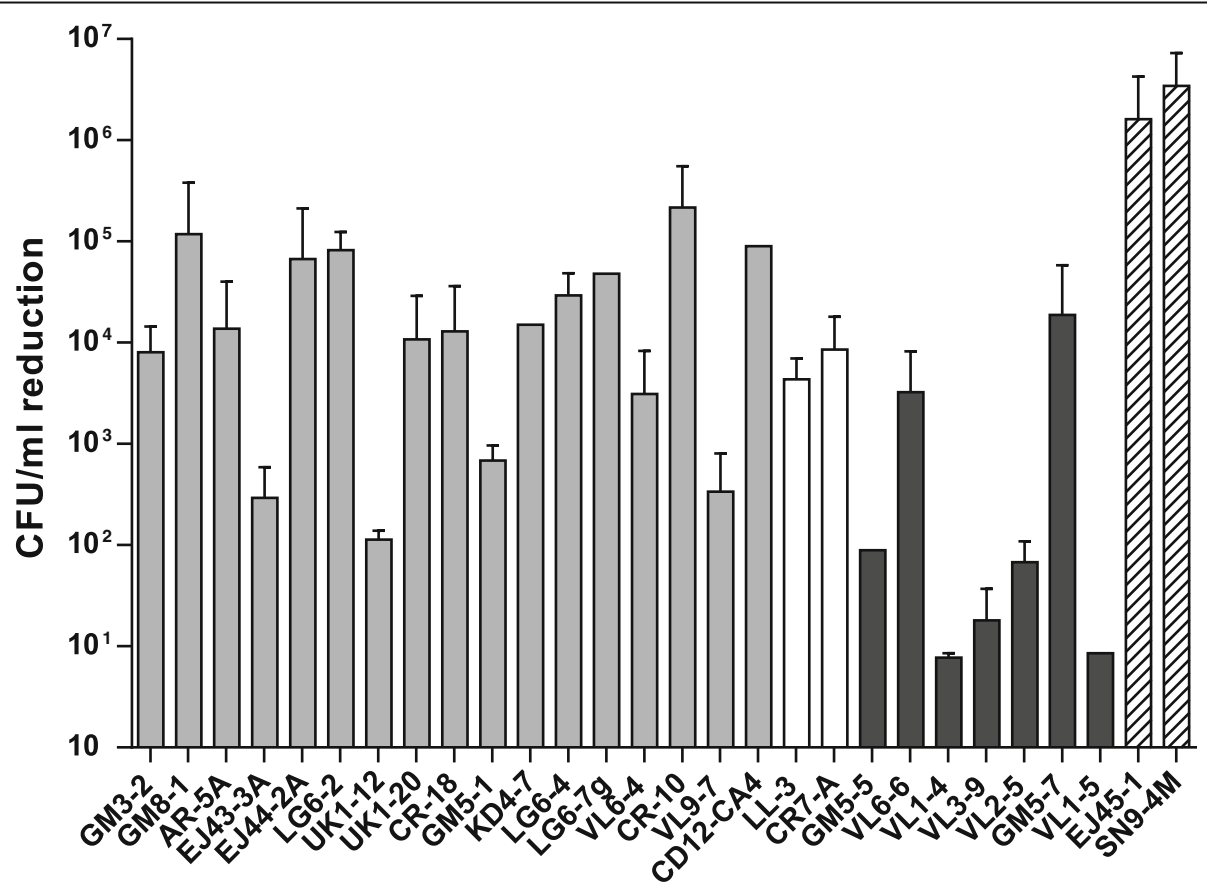

Fig. 2 Serum resistance assay showed diverse CFU reduction among the nasal Moraxella spp. strains. Survival of Moraxella pluranimalium strains (gray bars), uncertainly classified Moraxella strains (white bars), strains from the putative new Moraxella species cluster (black bars) and M. porci (streaked bars) was tested with $90 \%$ rabbit serum. Initial bacterial inoculum was approximately $10^{6} \mathrm{CFU} / \mathrm{ml}$. Results are expressed as average of the log CFU reduction after $1 \mathrm{~h}$ of incubation at $37^{\circ} \mathrm{C}$ with the serum, error bars indicate standard deviation from three replicate assays 


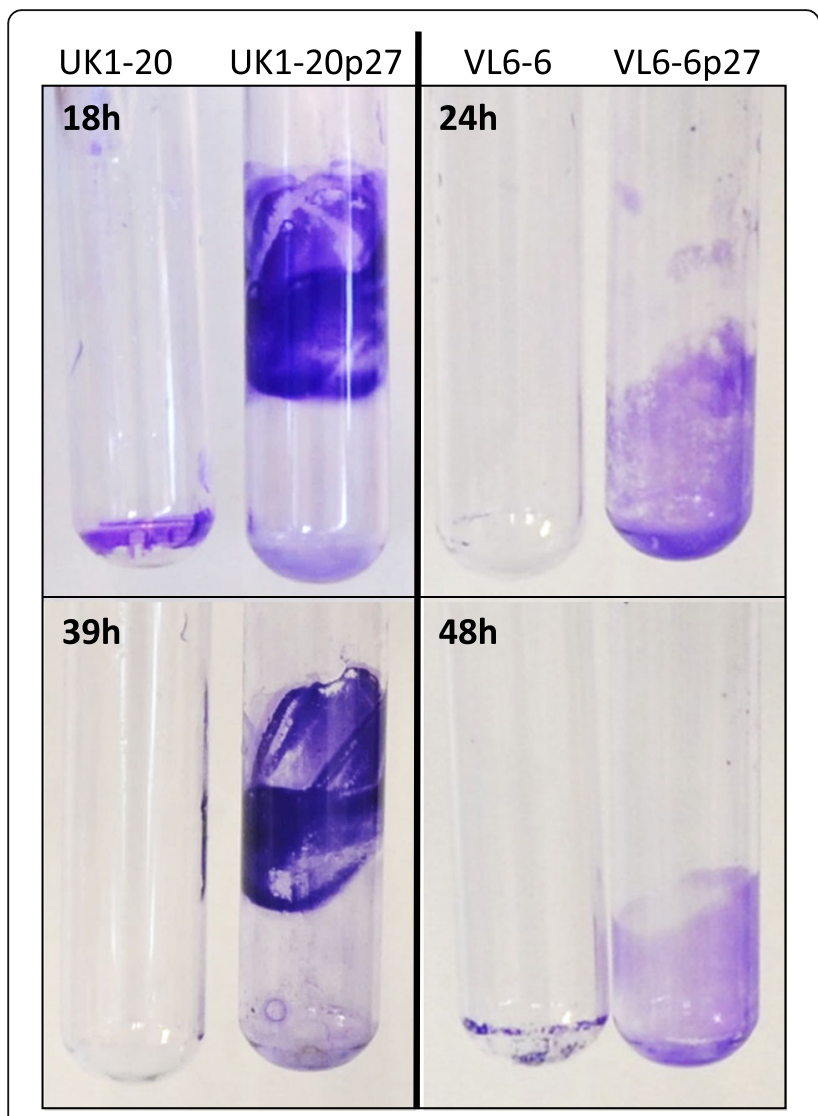

Fig. 3 Effect of culture passages on biofilm formation. Biofilm produced in glass tubes by strains UK1-20 and VL6-6 after 27 culture passages in the laboratory (UK1-20p27 and VL6-6p27). The original strains are included in the pictures for comparison. Cultures were incubated for different times (indicated in the figure) and pictures were taken after elimination of the bacterial culture and staining with crystal violet. Although it is not clear in the picture, VL6-6p27 formed biofilm as a floating pellicle at $24 \mathrm{~h}$

level. Under static conditions, we observed a common trend of decreasing biofilm production when comparing 24 and $48 \mathrm{~h}$. In contrast, we observed the opposite effect under shaking conditions, with most of the strains showing a tendency of increasing biofilm production with time. Strains with slow growth (unclassified cluster) showed the same trend in decreasing biofilm production under both conditions. Biofilm formation was not affected by precoating the wells with mucin or bovine serum albumin (BSA) (data not shown).

\section{Cell adhesion}

To explore whether the biofilm formation was associated with cell adhesion capacity, selected strains with different rates of biofilm formation were assayed in PK-15 and A549 cell lines. Although the assayed strains presented adhesion ability to cells, the observed differences in biofilm formation capacity did not correlated with differences in cell adhesion to both PK-15 (not shown) or A549 cells (Fig. 5).

\section{Association to porcine alveolar macrophages}

Selected strains representing the diversity in phylogenetic, serum resistance and biofilm formation were tested with porcine alveolar macrophages (PAMs). Different levels of association with PAMs were observed among the strains (Fig. 6). Within the 4 Moraxella pluranimalium strains tested, only LG6-2 presented a clear association with PAMS, similar to the level observed in the susceptible $H$. parasuis strain used as control. Moraxella porci EJ45-1, unlike the $M$. porci type strain SN9-4M, showed high levels of association with PAMs. The three isolates from the putative new species cluster presented low association with macrophages, even lower than the $H$. parasuis phagocytosis resistant strain used as control (Fig. 6). Levels of association to macrophages were not directly linked to biofilm formation capacity or serum resistance (Table 3).

\section{In vitro culture passages affected association to macrophages}

In vitro passages affected the autoagglutination and biofilm formation capacity of $M$. plurianimalium UK1-20 and Moraxella sp. VL6-6 as indicated above. Although enhanced adhesion to epithelial cells was not observed in UK1-20p27 (not shown), this strain showed higher association with PAMs than the original strain ( $\mathrm{T}$ test, $P=0.04$; Fig. 6). In contrast, VL6-6p27 showed the same level of interaction with PAMs as the original strain VL6-6 (Fig. 6); this may be due to a different adaptation to laboratory conditions, as observed by the different type of biofilm formed by this strain.

\section{Discussion}

Moraxella spp. from the nasal microbiota of piglets showed heterogeneous characteristics, from antimicrobial resistance profiles to virulent mechanisms and adherence properties. The majority of the isolates belonged to $M$. plurianimalium species, but we also detected $M$. porci, a couple of isolates of uncertain classification and a cluster of strains that may constitute a new Moraxella species. This cluster comprised strains with a slower growth rate in broth than the rest of the nasal strains and contained most of the strains with high resistance to the serum complement.

Higher rates of antimicrobial resistance were found in strains from farms undergoing antimicrobial treatments, but further studies are needed to demonstrate that the administration of these drugs promotes resistance in this bacterial genus. MDR strains were broadly detected, and only one strain, UK1-20, from a farm where no antibiotics were used, did not show resistance to any of the 

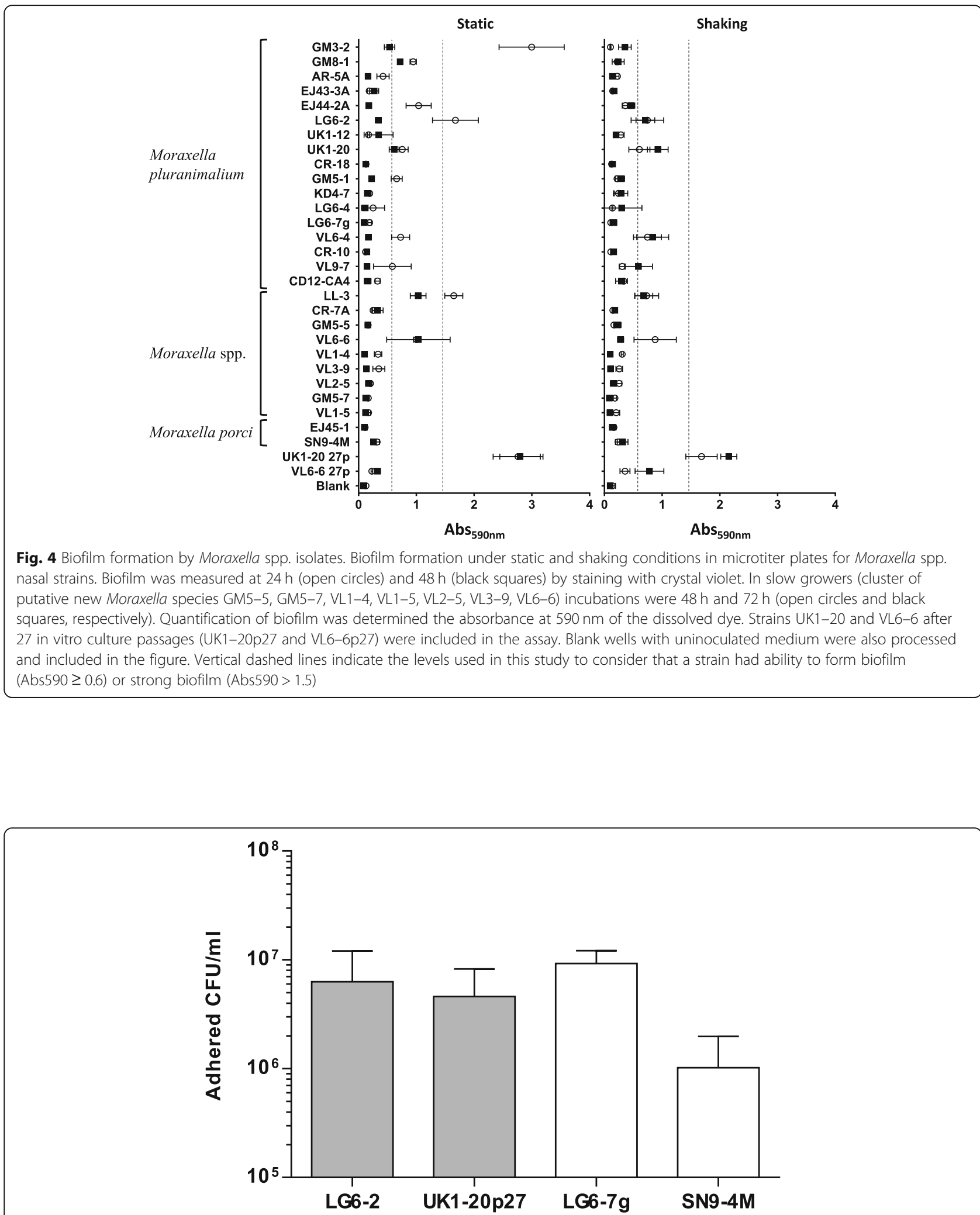

Fig. 5 Bacteria adhesion to A549 cells. Two biofilm forming strains, LG6-2 and UK1-20p27, and two non-forming strains, LG6-7 g and SN9-4 M, were incubated with pulmonary A549 cells to examine their adhesion capacity. After $1 \mathrm{~h}$ of incubation, non-attached bacteria were washed and attached bacteria were quantify by dilutions and plating. Results represent the average adherent bacteria from 3 different assays (error bars: standard deviation) 


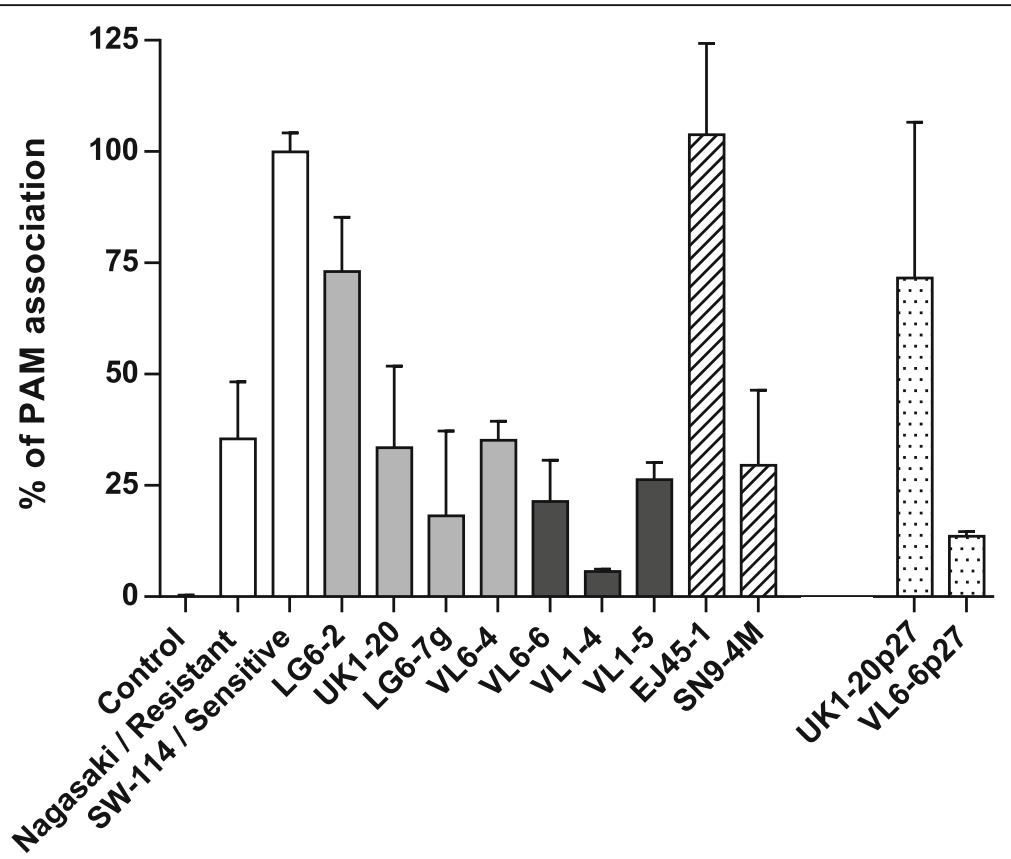

Fig. 6 Phagocytosis susceptibility of selected Moraxella spp. strains. Selected M. pluranimalium strains (gray bars), strains from the putative new species cluster (black bars) and M. porci strains (streaked bars). FITC-labelled Moraxella spp. strains were incubated with porcine alveolar macrophages (PAMs) during $1 \mathrm{~h}$ at $37^{\circ} \mathrm{C}$. After washing to eliminate unbound bacteria, association of bacteria with PAMs was measured by flow cytometry and expressed as \% of fluorescent PAMs. H. parasuis susceptible strain SW114 and resistant strain Nagasaki were included as control for the assay (white bars) and SW114 levels of phagocytosis were used to normalize the results of different tests. In addition, strains UK1-20 and VL6-6 after 27 passages on agar plates were also tested (dotted bars). Results represent the average of duplicate wells from 2 different assays (error bars: standard deviation)

Table 3 Summary of results from serum susceptibility, association to alveolar macrophages and biofilm formation capacity for selected Moraxella spp. Isolates

\begin{tabular}{|c|c|c|c|}
\hline Strains & Biofilm formation ${ }^{b}$ & Serum susceptibility ${ }^{c}$ & $\begin{array}{l}\text { Association to } \\
\text { macrophages }\end{array}$ \\
\hline \multicolumn{4}{|c|}{ M. pluranimalium } \\
\hline LG6-2 & + & $\mathrm{S}$ & S \\
\hline UK1-20 & + & S & $\mathrm{R}$ \\
\hline LG6-7 g & - & $\mathrm{S}$ & $\mathrm{R}$ \\
\hline VL6-4 & + & S & $\mathrm{R}$ \\
\hline \multicolumn{4}{|c|}{ Moraxella sp. ${ }^{a}$} \\
\hline VL6-6 & + & $\mathrm{S}$ & $\mathrm{R}$ \\
\hline VL1-4 & - & $\mathrm{R}$ & $\mathrm{R}$ \\
\hline $\mathrm{VL} 1-5$ & - & $\mathrm{R}$ & $\mathrm{R}$ \\
\hline \multicolumn{4}{|l|}{ M. porci } \\
\hline EJ45-1 & - & S & S \\
\hline SN9-4 M & - & S & $\mathrm{R}$ \\
\hline
\end{tabular}

${ }^{\mathrm{a}}$ Strains in the putative new Moraxella species cluster

${ }^{\mathrm{b}}$ Abs590 $>0.8$ in the crystal violet assay

'Viability reduction of more than 3 logarithms antimicrobials tested. It is important to highlight the presence of four MDR strains of Moraxella pluranimalium, with a high number of drug resistances, including colistin in two of them. This latter observation is consistent with the recent discovery of colistin resistance genes in Moraxella [10-12]. Coding genes of antibiotic resistances can be horizontally transferred between bacteria of the same or even different species, and therefore, the presence of resistance genes in the resident microbiota is of concern since they could be transferred to potential pathogens [13]. Diversity of the nasal Moraxella isolates was also evident in the in vitro assays, where strains showed a wide heterogeneity.

Biofilms are traditionally considered forms of resistance to environmental conditions and a virulence mechanism in bacteria of clinical importance [14]. As for other characteristics, we observed heterogeneity in biofilm formation capacity of the porcine nasal strains of Moraxella. Variability in biofilm formation has been already reported for other species of Moraxella from ruminants [15]. Biofilm formation capacity can be associated also to the colonizing ability of the bacteria, as was observed for $H$. parasuis [16]. The interaction of the nasal Moraxella strains with the respiratory mucosa needs more study, but attachment to mucin as wells as to epithelial cells could play a role, as suggested by our results. 
On the other hand, bacteria from the upper respiratory tract can occasionally reach the lower tract, where they will be confronted with other components of the immune system, such as the alveolar macrophages. In vitro assays with PAMs provided insight into the potential of the nasal isolates to survive this first barrier of cell immunity in the lung. Heterogeneity in this feature has been shown for other bacterial species, such as $H$. parasuis [17] or Klebsiella pneumoniae [18]. In our case, strains with high association to PAMs, such as LG6-2, were observed, while other strains did not associate with the phagocytic cells, as the case of the virulent SN9-4 M. According to the results, macrophage association is not directly associated with the phylogeny of the isolates, since heterogeneity in this trait was observed within the distinct species or clusters. One paradigmatic case could be EJ45-1 and SN9-4 M from M. porci, having high differences between them. The importance that phagocytosis resistance could have for in vivo infection/colonization by M. pluranimalium is supported by the fact that originally the UK1-20 strain showed no association with PAMs (indicative of resistance to phagocytosis), but lost this trait after in vitro culture passages, in agreement with the loss of virulence after laboratory adaptation in other bacteria [19]. Loss of phagocytosis resistance in UK1-20 was concurrent with increase biofilm formation capacity. Thus, biofilm formation may be relevant in environmental persistence, whereas phagocytosis resistance will be important during host infection. However, this phenomenon seems to be strain-dependent, since it was not observed with strain VL6-6, which did not show an adaptation to laboratory conditions that affected its original resistance to PAMs.

Most of the assayed Moraxella spp. strains showed sensitivity to the serum complement, with some exceptions, including most of the strains in the cluster representing a putative new species. Thus, most nasal Moraxella strains are probably poorly invasive and will be kept on the nasal mucosa, but the new putative species cluster deserves more attention to define their role in disease in pigs.

\section{Conclusions}

The characterization of Moraxella spp. isolates from the nasal cavities of young piglets demonstrated a high heterogeneity within this genus. Most of the isolates were identified as $M$. pluranimalium, and only one as $M$. porci. A group of 7 isolates clustered together by $16 \mathrm{~S}$ rRNA gene sequence and did not show homology to any known Moraxella species. Many of the Moraxella spp. strains were resistant to multiple antimicrobial classes. Heterogeneity of the Moraxella strains was also evident in adhesion and virulence-associated assays. Further analyses, including experimental infections, need to be done to explain the function of this genus in swine health and disease.

\section{Methods \\ Isolation and bacteria cultures}

Nasal cavities from 10 piglets of around 3 weeks of age from 5 different litters were sampled from 8 commercial farms (Table 1) with standard management practices for husbandry and welfare of the animals. Sampling of piglets was done under institutional authorization and followed good veterinary practices. According to European (Directive 2010/63/EU of the European Parliament and of the Council of 22 September 2010 on the protection of animals used for scientific purposes) and Spanish (Real Decreto 53/2013) normative, this procedure did not require specific approval by an Ethical Committee. Nasal sampling was performed only once to each piglet and is not likely to cause pain, suffering, distress or lasting harm equivalent to, or higher than, that caused by the introduction of a needle in accordance with good veterinary practice (Chapter I, Article 1, 5 (f) of 2010/63/EU). Nasal swabs were transported in Amies medium to the laboratory, where they were plated on chocolate agar (Biomérieux, Marcy l'Étoile, France) to isolate colonies. After 24 and $48 \mathrm{~h}$ of incubation at $37{ }^{\circ} \mathrm{C}$ and $5 \% \mathrm{CO}_{2}$, different colonies were selected and stored at $-80{ }^{\circ} \mathrm{C}$ in $20 \%$ glycerol-Brain Heart Infusion broth (BHI) for further characterization. Bacterial suspensions were also performed in phosphate-buffered saline (PBS) for DNA extraction.

In addition, reference strains of Moraxella porci SN9$4 \mathrm{M}$ and Moraxella pluranimalium CD12-CA4, both isolated from lesions of diseased pigs, were used in this study. For assays with PAMs, 2 reference strains of Haemophilus parasuis were used as controls.

\section{Bacterial identification and genotyping}

DNA extraction was performed using Chelex based Instagene $^{\mathrm{Tu}}$ Matrix (Bio-Rad Laboratories, Hercules, CA, USA) following manufacturer's instructions. Preliminary identification of isolates was performed by partial sequencing of the 16S rRNA gene using primers 358F (CTACGGGAGGCAGCAGT) and 907R (CCGTCWATTCMTTTGAGTTT) [20]. Sequences were analyzed by blasting against the Ribosomal database (http://rdp.cme.msu.edu).

All isolates identified as Moraxella were then genotyped by ERIC-PCR [21] with primers ERIC-1F (ATGTAAGCTC CTGGGGATTCAC) and ERIC-2R (AAGTAAGTGACTG GGGTGAGCG). PCR reaction mixture consisted of $3 \mathrm{mM}$ of $\mathrm{MgCl}_{2}, 1.2 \mu \mathrm{M}$ of each primer, $0.23 \mathrm{mM}$ of dNTPs, 0.75 $\mathrm{U}$ of GoTaq polymerase (Promega, Madison Wisconsin, USA) and 100 ng of DNA sample. Amplification was carried out with an initial denaturation of $94^{\circ} \mathrm{C}$ for 2 min followed by 30 cycles of $30 \mathrm{~s}$ at $94{ }^{\circ} \mathrm{C}, 1 \mathrm{~min}$ at $50^{\circ} \mathrm{C}$ and $2.5 \mathrm{~min}$ at $72{ }^{\circ} \mathrm{C}$, and a final extension of $20 \mathrm{~min}$ at $72^{\circ} \mathrm{C}$. One isolate 
from each fingerprinting profile was selected for further analysis.

Final identification of the different Moraxella strains was performed by $16 \mathrm{~S}$ rRNA gene amplification with universal primers 8F (AGAGTTTGATCCTGGCTCAG) and 1492R (CGGTTACCTTGTTACGACTT) [22] and sequencing with 8 F, 1492R, 358F and 907R primers. Sequence analysis was performed with Fingerprinting II v3.0 software (BioRad) and a UPGMA dendrogram was built for species assignment, including sequences from different Moraxella spp. type strains. Biochemical characterization of representative isolates was performed using the Vitek 2 system (Biomerieux).

\section{Antimicrobial susceptibility}

Susceptibility to several antimicrobials was tested as previously described [23]. Neo-Sensitabs ${ }^{\mathrm{Tm}}$ diffusion tablets (Rosco Diagnostica, Taastrup, Denmark) were used for Gentamicin, Ceftiofur, Colistin, Erythromycin, Lincoespectin, Tetracycline, Doxycycline, Trimetoprim + Sulfamide $(\mathrm{T}+\mathrm{S})$, Enrofloxacin, Amoxicillin and Amoxicillin + Clavulanic acid testing. Marbofloxacin, florfenicol and tulathromycin were tested in chocolate agar plates at different dilutions, from 16 to $0.125 \mu \mathrm{g} / \mathrm{mL}$ in the case of florfenicol and marbofloxacin; and from 64 to $0.125 \mu \mathrm{g} /$ $\mathrm{mL}$ in the case of tulathromycin. McFarland suspensions of 0.5 in $0.9 \% \mathrm{NaCl}$ were prepared for each strain and streaked on the plates with the different antibiotic concentration. Minimal inhibitory concentration (MIC) was determined observing the bacterial growth after $24 \mathrm{~h}$ of incubation. As no clinical breakpoints are available for these species, available CLSI breakpoints for Moraxella catarrhalis were taken for Tetracycline, Doxycycline, Trimetoprim+Sulfonamide, and veterinary practice according to CLSI breakpoints was followed for the rest of drugs (EUCAST-and CLSI potency NEO-SENSITABS, Rosco Diagnostica, 2013).

\section{Serum resistance assay}

Serum resistance assay was carried out with rabbit serum (EU Directive 2010/63/EU and Spanish normative Real Decreto 53/2013 were followed). A bacterial suspension of each strain (representative of each ERIC fingerprinting) was prepared in PBS with $20 \%$ glycerol to reach an $\mathrm{OD}_{600}$ of 0.3 in a VIS 7200 spectrophotometer (Dinko Instruments, Barcelona, Spain). In duplicate wells, $10 \mu \mathrm{l}$ of the bacterial suspension (approx. $10^{6} \mathrm{col}-$ ony forming units $[\mathrm{CFU}] / \mathrm{mL}$ ) were mixed with $90 \mathrm{ul}$ of fresh filtered rabbit serum and mixtures were incubated for $1 \mathrm{~h}$ at $37^{\circ} \mathrm{C}$ and $100 \mathrm{rpm}$. Bacterial survival was calculated by comparing bacterial counts (obtained by serial dilutions and plating) at time 0 and after $1 \mathrm{~h}$ incubation. The assay was carried out 3 times for each strain.

\section{Association to porcine alveolar macrophages}

Association to macrophages was tested as previously described with PAMs [17]. PAMs were obtained from healthy piglets euthanized by intravenous pentobarbital overdose under institutional authorization. All procedures involving animals followed EU and Spanish normative (Directive 2010/63/EU and Real Decreto 53/ 2013). Lungs were removed and PAMs were isolated by lung lavage with PBS containing $70 \mu \mathrm{g} / \mathrm{mL}$ of gentamicin. PAMs were collected by centrifugation at $241 \times \mathrm{g}$ for $15 \mathrm{~min}$, washed twice with sterile PBS and stored at $-150^{\circ} \mathrm{C}$ in $10 \%$ Dimethylsulfoxide (DMSO) in fetal bovine serum (FBS) until use.

Selected nasal strains of Moraxella were tested to determine their level of association to PAMs. Moraxella porci reference strain SN9-4 M was also included in the assay. In addition of two reference strains of Haemophilus parasuis, Nagasaki (virulent) and SW-114 (non-virulent), were included as control for the technique.

For the assay, PAMs were plated in Dulbecco's Modified Eagle Medium (DMEM) supplemented with 10\% of FBS and $1 \%$ glutamine at a concentration of $5 \times 10^{5}$ cells per well in 6 well-plates. Plates were incubated 1-2 h to allow the attachment of the macrophages to the bottom of the wells. For each strain duplicate wells were inoculated with $10^{7} \mathrm{CFU}$ of fluorescein isothiocyanate (FITC)labelled bacteria (bacterial inocula were confirmed by dilutions and plating). After $1 \mathrm{~h}$ of incubation at $37^{\circ} \mathrm{C}$ and $5 \% \mathrm{CO}_{2}$, plates were transferred to an ice bath to stop interaction and were washed twice with PBS to eliminate unbound bacteria. PAMs were then scrapped in PBS and were analyzed by flow cytometry using an EPICS XL-MCLTM Flow Cytometer (Beckman Coulter, Madrid, Spain) or MACSQuant Analyzer 10 (Myltenyi Biotec, Bergisch Gladbach, Germany). Assays were repeated with PAMs from different animals that were already available at the laboratory. Since each batch of macrophages showed different level of activity, values were harmonized considering the reference $H$. parasuis strain SW114 (phagocytosis sensitive) as 100\% phagocytosis in each assay; i.e., using the following calculation: (\% PAMs with test bacteria - \% fluorescence background in PAMs without bacteria / \% PAMs with positive control SW114 - \% fluorescence background in PAMs without bacteria) $\times 100$.

\section{Biofilm assays}

Biofilm assays were performed in 96 well cell culture plates under static and shaking conditions following previous published protocol with some modifications (Bello-Ortí et al. 2014). Bacterial suspensions were made to reach an $\mathrm{OD}_{600} 0.3$ in BHI. Wells in 96 well plates were then inoculated in quadruplicate with a 1:100 dilution of the bacterial suspension in BHI. Plates were 
incubated at $37^{\circ} \mathrm{C}$, under static conditions with $5 \% \mathrm{CO}_{2}$ or under agitation at $100 \mathrm{rpm}$ for 24 and $48 \mathrm{~h}$, except for slow growing isolates, which were incubated for 48 and $72 \mathrm{~h}$. After incubation, wells were emptied and rinsed with tap water to remove unattached bacteria. Wells were then stained with $0.1 \%(\mathrm{w} / \mathrm{v})$ crystal violet (Merck, Darmstadt, Germany) for $2 \mathrm{~min}$ at room temperature. Wells were washed thrice with tap water to remove the excess of crystal violet and let dried at $37{ }^{\circ} \mathrm{C}$. The dye in the stained biofilms was solubilized with $100 \mu \mathrm{L}$ of $70 \%$ ethanol and quantified at $590 \mathrm{~nm}$ in a Powerwave XS Microplate Spectrophotometer (Biotek Instruments Inc., Winooski, VT, USA). Biofilm formation was also assessed on wells pre-coated with $5 \mu \mathrm{g}$ of mucin or BSA as control.

\section{Cell adhesion assay}

Porcine epithelial cells PK-15 (ATCC ${ }^{\oplus}$ CCL-33) and human lung A549 (ATCC ${ }^{\oplus}$ CCL-185) cell lines were cultured in DMEM supplemented with 1\% glutamine and 5\% FBS for PK-15 and 10\% for A549 cells.

Concentration of cells per $\mathrm{ml}$ was calculated to obtain a monolayer after overnight incubation, approximately 50,000 cells/well in cell culture 96 well plates. After overnight incubation, wells with PK-15 or A549 cells were washed once with sterile PBS. Duplicate wells were inoculated with $10^{7} \mathrm{CFU} / \mathrm{mL}$ bacteria from each isolate tested. Bacterial inoculum quantification was confirmed by colony counts. Microplates were then centrifuged 10 min at $100 \times g$ to facilitate the contact between bacteria and cells. After incubation for $1 \mathrm{~h}$ at $37^{\circ} \mathrm{C}$ and $5 \% \mathrm{CO}_{2}$, wells were washed twice with sterile PBS to remove unattached bacteria, and attached bacteria were released with $0.1 \%$ of saponin and pipetting. The resulting suspension was quantified by plating dilutions on agar plates.

\section{Supplementary information}

Supplementary information accompanies this paper at https://doi.org/10. 1186/s12917-020-2250-9.

Additional file 1. Biochemical characterization by Vitek 2 (Biomerieux) of representative strains.

\section{Abbreviations}

BHI: Brain Heart Infusion; BSA: Bovine serum albumin; CFU: Colony forming units; DMEM: Dulbecco's Modified Eagle Medium; DMSO: Dimethylsulfoxide; ERIC: Enterobacterial repetitive intergenic consensus; FBS: Fetal bovine serum; FITC: Fluorescein isothiocyanate; MDR: Multidrug-resistant; MIC: Minimal inhibitory concentration; PAMs: Porcine alveolar macrophages; UPGMA: Unweighted pair group method with arithmetic mean

\section{Acknowledgements}

A preliminary version of this study was presented at the Prato Conference on the Pathogenesis of Bacterial Infections of Animals 2016, as a poster presentation (http://vetpath-2016.p.asnevents.com.au/days/2016-10-12/ abstract/38673).

\section{Authors' contributions}

SLS performed and analyzed the experiments and drafted the manuscript, NGM, MCH and AMPR performed experiments, and VA conceived the study, analyzed results and helped to draft the manuscript. All authors have read and approved the manuscript.

\section{Funding}

This work was supported by the Ministry of Economy and Competitiveness (MINECO) of the Spanish Government (grant numbers AGL2013-45662 and AGL2016-77361). MCH is a recipient of a Juan de la Cierva fellowship, from MINECO (Spain). The authors are also grateful to the Centres de Recerca de Catalunya (CERCA) Programme. Funding institutions did not influence this study.

\section{Availability of data and materials}

The datasets analysed during the current study are available from the corresponding author on reasonable request.

\section{Ethics approval and consent to participate}

Verbal consent for sampling from the owners of the animals was obtained, following common practice for this type of sampling. Piglet sampling was part of routine veterinary practice in the farms. Sampling of piglets was done under institutional authorization and followed good veterinary practices. According to European (Directive 2010/63/EU of the European Parliament and of the Council of 22 September 2010 on the protection of animals used for scientific purposes) and Spanish (Real Decreto 53/2013) normative, this procedure did not require specific approval by an Ethical Committee. Nasal sampling was performed only once to each piglet and is not likely to cause pain, suffering, distress or lasting harm equivalent to, or higher than, that caused by the introduction of a needle in accordance with good veterinary practice (Chapter I, Article 1, 5 ( $f$ of 2010/63/EU). Blood sampling of rabbit to obtain serum was performed following good veterinary practices and the above mentioned normative. For PAMs, following Article 18 of 2010/63/EU (Sharing organs and tissues), lungs were obtained from animals killed for other reasons.

\section{Consent for publication}

Not applicable.

\section{Competing interests}

The authors declare that they have no competing interests. None of the authors has any financial or personal relationships that could inappropriately influence or bias the content of the paper.

Received: 6 November 2019 Accepted: 20 January 2020

Published online: 30 January 2020

References

1. Verduin $\mathrm{CM}, \mathrm{Hol} \mathrm{C}$, Fleer A, van Dijk H, van Belkum A. Moraxella catarrhalis: from emerging to established pathogen. Clin Microbiol Rev. 2002;15:125-44.

2. Postma GC, Carfagnini JC, Minatel L. Moraxella bovis pathogenicity: an update. Comp Immunol Microbiol Infect Dis. 2008;31:449-58.

3. Vela Al, Sanchez-Porro C, Aragon V, Olvera A, Dominguez L, Ventosa A, Fernandez-Garayzabal JF. Moraxella porci sp. nov., isolated from pigs. Int J Syst Evol Microbiol. 2010;60:2446-50.

4. Vela Al, Arroyo E, Aragon V, Sanchez-Porro C, Latre MV, Cerda-Cuellar M, Ventosa A, Dominguez L, Fernandez-Garayzabal JF. Moraxella pluranimalium sp. nov., isolated from animal specimens. Int J Syst Evol Microbiol. 2009;59:671-4.

5. Correa-Fiz F, Fraile L, Aragon V. Piglet nasal microbiota at weaning may influence the development of Glasser's disease during the rearing period. BMC Genomics. 2016;17:404

6. Weese JS, Slifierz M, Jalali M, Friendship R. Evaluation of the nasal microbiota in slaughter-age pigs and the impact on nasal methicillinresistant Staphylococcus aureus (MRSA) carriage. BMC Vet Res. 2014;10:69.

7. Cormier Y, Tremblay G, Meriaux A, Brochu G, Lavoie J. Airborne microbial contents in two types of swine confinement buildings in Quebec. Am Ind Hyg Assoc J. 1990;51:304-9.

8. Fung TC, Olson CA, Hsiao EY. Interactions between the microbiota, immune and nervous systems in health and disease. Nat Neurosci. 2017;20:145-55. 
9. Biesbroek G, Tsivtsivadze E, Sanders EA, Montijn R, Veenhoven RH, Keijser BJ Bogaert D. Early respiratory microbiota composition determines bacterial succession patterns and respiratory health in children. Am J Respir Crit Care Med. 2014:190:1283-92.

10. AbuOun M, Stubberfield EJ, Duggett NA, Kirchner M, Dormer L, NunezGarcia J, Randall LP, Lemma F, Crook DW, Teale C, Smith RP, Anjum MF. $m c r-1$ and mcr-2 (mcr-6.1) variant genes identified in Moraxella species isolated from pigs in Great Britain from 2014 to 2015. J Antimicrob Chemother. 2017;72:2745-9.

11. Poirel L, Kieffer N, Fernandez-Garayzabal JF, Vela Al, Larpin Y, Nordmann P. MCR-2-mediated plasmid-borne polymyxin resistance most likely originates from Moraxella pluranimalium. J Antimicrob Chemother. 2017;72:2947-9.

12. Kieffer N, Nordmann P, Poirel L. Moraxella Species as Potential Sources of MCR-Like Polymyxin Resistance Determinants. Antimicrob Agents Chemother. 2017;61:e00129-17.

13. Penders J, Stobberingh EE, Savelkoul PH, Wolffs PF. The human microbiome as a reservoir of antimicrobial resistance. Front Microbiol. 2013;4:87.

14. Hall-Stoodley L, Costerton JW, Stoodley P. Bacterial biofilms: from the natural environment to infectious diseases. Nat Rev Microbiol. 2004;2:95-108.

15. Ely VL, Vargas AC, Costa MM, Oliveira HP, Potter L, Reghelin MA, Fernandes AW, Pereira DIB, Sangioni LA, Botton SA. Moraxella bovis, Moraxella ovis and Moraxella bovoculi: biofilm formation and lysozyme activity. J Appl Microbiol. 2019;126:369-76.

16. Bello-Orti B, Deslandes V, Tremblay YD, Labrie J, Howell K, Tucker AW, Maskell DJ, Aragon V, Jacques M. Biofilm formation by virulent and nonvirulent strains of Haemophilus parasuis. Vet Res. 2014:45:104.

17. Olvera A, Ballester M, Nofrarias M, Sibila M, Aragon V. Differences in phagocytosis susceptibility in Haemophilus parasuis strains. Vet Res. 2009;40:24.

18. Cavalcanti FCN, Rodrigues JF, Cabral AB, Azarias LCB, Morais Junior MA, Castro C, Lopes ACS. Relationships between phagocytosis, mucoid phenotype, and genetic characteristics of Klebsiella pneumoniae clinical isolates. Rev Soc Bras Med Trop. 2019;52:e20190089B.

19. Gaillard ME, Bottero D, Castuma CE, Basile LA, Hozbor D. Laboratory adaptation of Bordetella pertussis is associated with the loss of type three secretion system functionality. Infect Immun. 2011;79:3677-82.

20. Lane DJ. 16S/23S rRNA sequencing. In: Stackebrandt E, Goodfellow M, editors. Nucleic acid techniques in bacterial systematics. England: Wiley; 1991. p. 115-65.

21. Versalovic J, Koeuth T, Lupski JR. Distribution of repetitive DNA sequences in eubacteria and application to fingerprinting of bacterial genomes. Nucleic Acids Res. 1991;19:6823-31.

22. Weisburg WG, Barns SM, Pelletier DA, Lane DJ. 165 ribosomal DNA amplification for phylogenetic study. J Bacteriol. 1991;173:697-703.

23. Lorenzo de Arriba M, Lopez-Serrano S, Galofre-Mila N, Aragon V. Characterisation of Bergeyella spp. isolated from the nasal cavities of piglets. Vet J. 2018;234:1-6.

\section{Publisher's Note}

Springer Nature remains neutral with regard to jurisdictional claims in published maps and institutional affiliations.

Ready to submit your research? Choose BMC and benefit from:

- fast, convenient online submission

- thorough peer review by experienced researchers in your field

- rapid publication on acceptance

- support for research data, including large and complex data types

- gold Open Access which fosters wider collaboration and increased citations

- maximum visibility for your research: over $100 \mathrm{M}$ website views per year

At $\mathrm{BMC}$, research is always in progress.

Learn more biomedcentral.com/submissions 